\title{
Inhalf
}

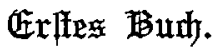 \\ Zngemeiner đeit.
}

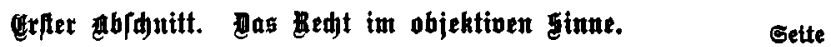

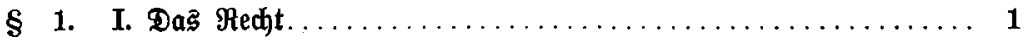

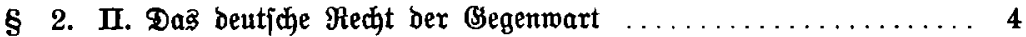

III. Das bürgerlidje materielle Recht.

§ 3. Die miffenidaftlidje Grappenbildung $\ldots \ldots \ldots \ldots \ldots \ldots$.

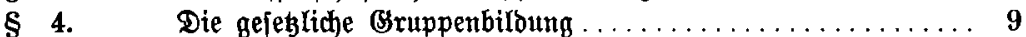

IV. Dả bürgerlidie (Befebbut(t).

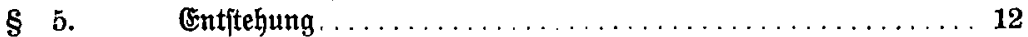

\& 6. Die $\mathfrak{R e b e n g e j e b e ~} \ldots \ldots \ldots \ldots \ldots \ldots \ldots \ldots \ldots \ldots, \ldots \ldots \ldots$

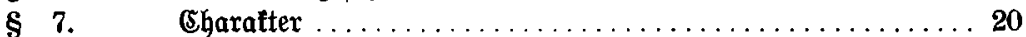

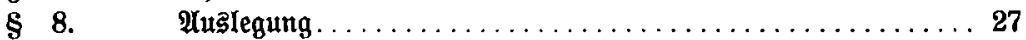

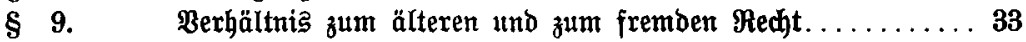

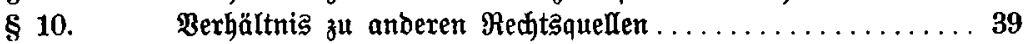

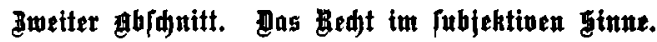

Erftes Rapitel. Grandlegung.

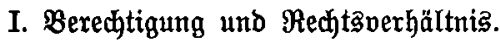

$\S 11 . \quad$ Der Brundbegriff $\ldots \ldots \ldots \ldots \ldots \ldots \ldots \ldots \ldots \ldots \ldots, 45$

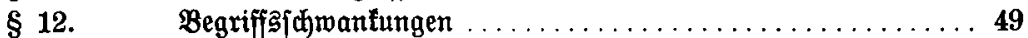

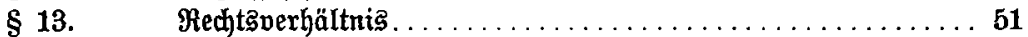

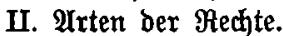

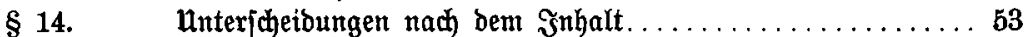

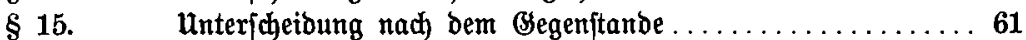

III. \$Beitere (3runbbegriffe.

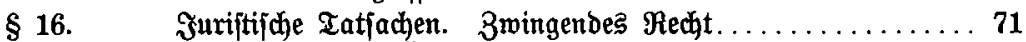

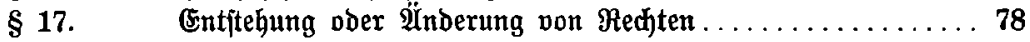

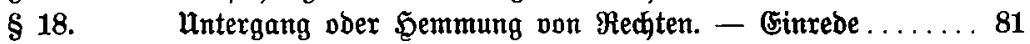

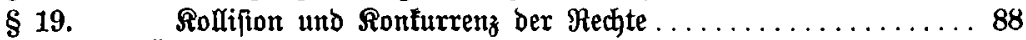

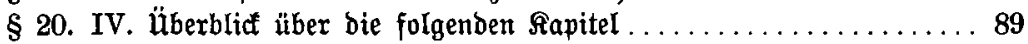

Zreites Rapitel. Die Redtsjubjette.

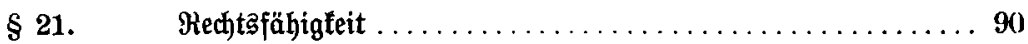

I. Die natürliden $\mathfrak{B e r j o n e n . ~}$ 
1. Borhandenjein und Arten.

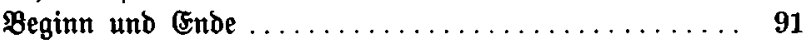

$\S 23$.

Bermutungen $\ldots \ldots \ldots \ldots \ldots \ldots \ldots \ldots \ldots \ldots \ldots \ldots, 92$

Irten ......................... 96

2. Perfönlichfeitşre孔te.

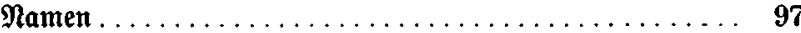

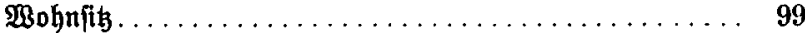

Perfönliche frreibeit. . . . . . . . . . . . . . . . 102

II. Die juriftijuen Berjonen.

§28. $\quad$ Begriffi und $\mathfrak{A r t e n} \ldots \ldots \ldots \ldots \ldots \ldots \ldots \ldots \ldots \ldots \ldots$

1. Rechtäfähige Bereine.

a) Bereine im allgemeinen

b) Singetragene Bereine.

c) $\mathfrak{U n d}$ ere rechţ̧ähige Bereine $\ldots \ldots \ldots \ldots \ldots \ldots \ldots \quad 123$

2. Stiftungen ...................... 125

Dritteg Rapitel. Die Redtzgegenftände.

I. Die Sadhen.

2. Unterfdjeidungen.

Wejentliche und unwejentliche Beftandteile.

Teilbare, unteilbare Sadhel................ 136

Rebenjađe, Zubebör ................... 136

$\S 42$.

II. Die Recte.

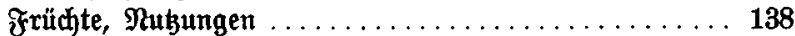

Dem $\mathfrak{B e r f e h r}$ entzogene Sadjen ............... 139

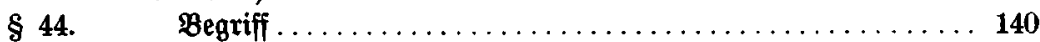

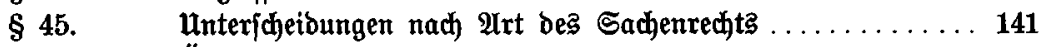

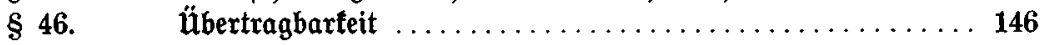

Biertes Rapitel. Die Ređtage ăäte.

I. Brunbbegriffe.

§ 47.

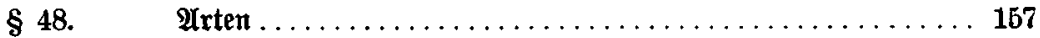

§ 49. Zwed, (Grund, Borausfebung $\ldots \ldots \ldots \ldots \ldots \ldots \ldots \ldots 161$

$\S 50 . \quad$ Bixffamfeit. . . . . . $\ldots \ldots \ldots \ldots \ldots \ldots \ldots \ldots \ldots$

II. Gelbịtänbige Sauptwillenserflärung.

1. Die einzelne $\mathfrak{B i l l e n ß e r f l a ̈ r u n g . ~}$

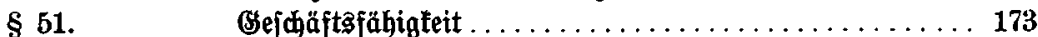

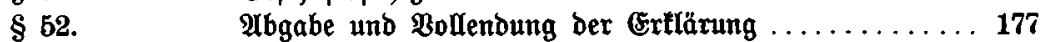

§ 53. $\quad$ Der Errłlärungßinbalt $\ldots \ldots \ldots \ldots \ldots \ldots \ldots \ldots \ldots \ldots$ 


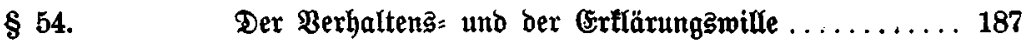

§55. Der Exfolgşmille uno ber gewünjhte Exfolg (\$rrtum,

Betrug und Drohung) ................... 189

§56. $\quad$ Ridjtigfteit und $\mathfrak{2 n}$ fechtbarfeit im einzelnen $\ldots \ldots \ldots \ldots 195$

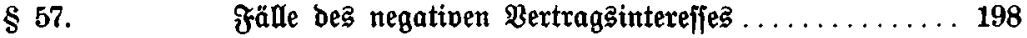

§58. Bedürfnis. Die Fälle eillfeitiger $\mathfrak{W i l l e n ß e r f l a ̈ r u n g ~ . ~ . ~ . ~} 199$

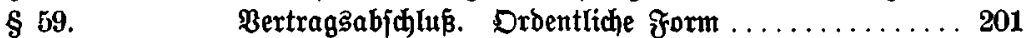

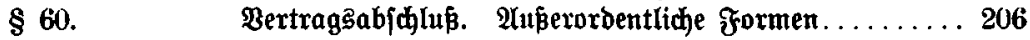

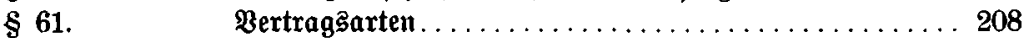

III. Crgänzungąbedürftige $\mathfrak{M i l l e n s e r f l a ̈ n u n g e n . ~}$

1. Steflpertretung.

\$ 62. $\mathfrak{B e g r i f f}$ und $\mathfrak{M o ̈ g l i d f e i t ~} \ldots \ldots \ldots \ldots \ldots \ldots \ldots \ldots 213$

\$ 63. Gewillürte Stellvertretuug auf (Srund von Bollmaゆt. 217

$\$ 64 . \quad$ Stelloertretung ohne Cinmilligung............. 220

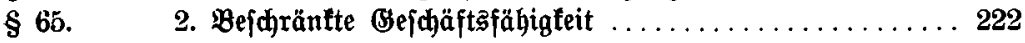

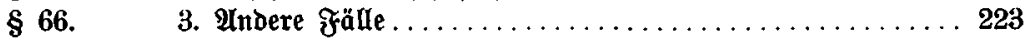

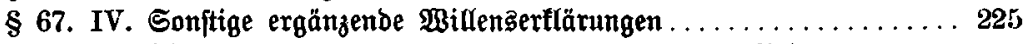

V. Cinzelne Inbalt3gejtaltungen von algemeiner Bebeutung.

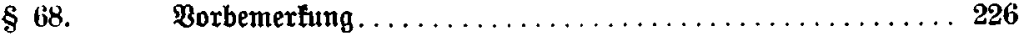

1. Bebingtes ßechtsgefdäft.

\$69. $\quad$ Begriff, $\mathfrak{2}$ rten, $\mathfrak{M o ̈ g l i c t l e i t ~} \ldots \ldots \ldots \ldots \ldots \ldots \ldots \ldots 226$

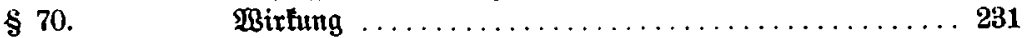

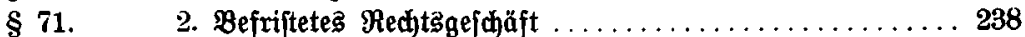

\$ 72. 3. \&iberalez $\Re$ Red

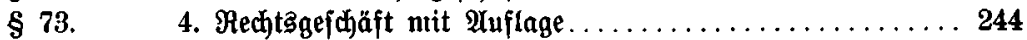

Fünftę Rapitel. $\mathfrak{B}$ erfdulden und Bertreten.

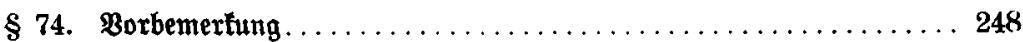

\$ 75. Schulbhaft rechtsmibriges $\mathfrak{B e r h a l t e n} \ldots \ldots \ldots \ldots \ldots \ldots \ldots \ldots 250$

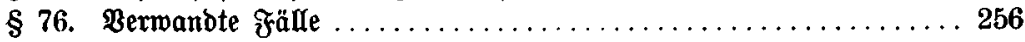

Sedstes Rapitel. Beitablauf.

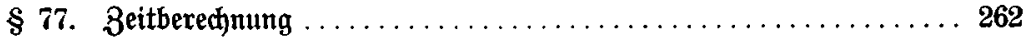

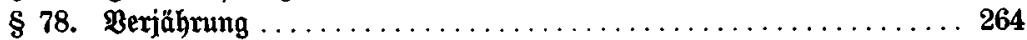

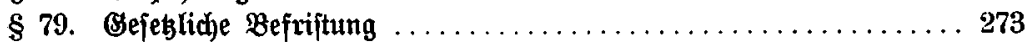

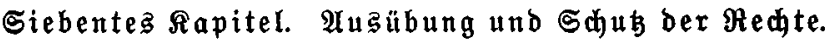

I. $\mathfrak{A}$ ußerprożefiual.

$\S 80 . \quad$ 1. $\mathfrak{I m}$ allgemeinen $\ldots \ldots \ldots \ldots \ldots \ldots \ldots \ldots \ldots \ldots \ldots \mathbf{2 7 4}$

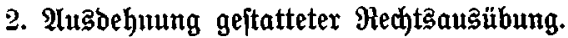

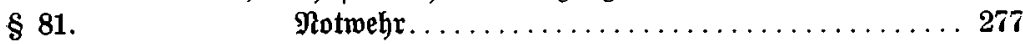

\$ 82. Notjtand, in abwehrenden Sinne.......... 279

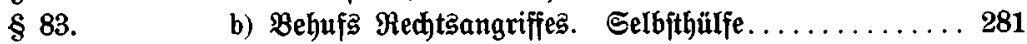

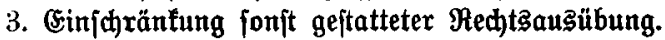

$\S 84 . \quad$ Notftand, im zugreifenden Sinne ............. 283

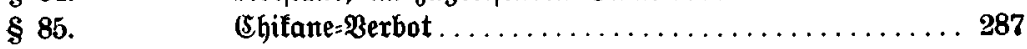


II. ßrozeffual.

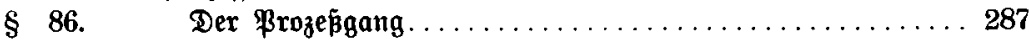

$\S 87 . \quad$ Beweig. . . . . . . . . . . . . . . . . . . . . . 290

$\S 88 . \quad$ Brozepbeginn und Ende $\ldots \ldots \ldots \ldots \ldots \ldots \ldots \ldots \ldots . \ldots \ldots$

Bmeife Bufy.

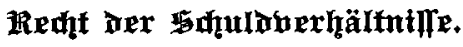

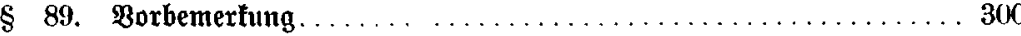

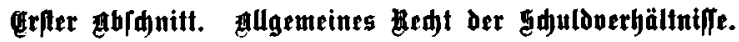

Erites Rapitel. Fubalt der Sduldoerhälniffe.

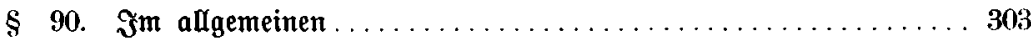

I. Die Эntenfität der Sdłulbverbältniffe.

$\$$ 91. 1. Berjönlide Leiftung und Bermögenshaftung ........ 305

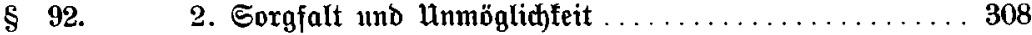

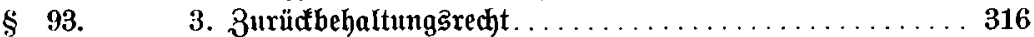

II. Der SSegenftand Der Sdulbverbältniffe.

$\$$ 94. 1. Cinige Einteilungen $\ldots \ldots \ldots \ldots \ldots \ldots \ldots \ldots \ldots \ldots \ldots$

2. Unbejtimmtheit de? Begenitandes.

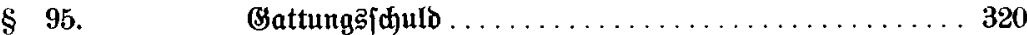

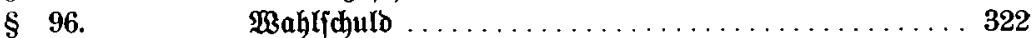

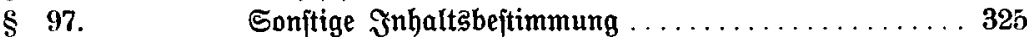

3. Einzelne bejondere Reiftungßgegenftände.

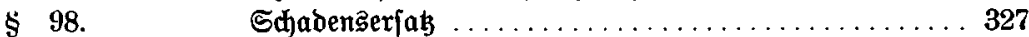

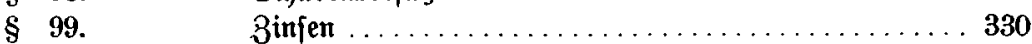

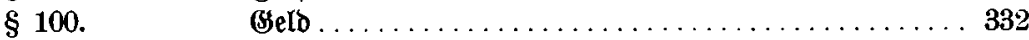

$\S 101 . \quad$ Sonjtige Reiftungsgegenftände $\ldots \ldots \ldots \ldots \ldots \ldots . \ldots 34$

III. Reiftungsort und 3eit.

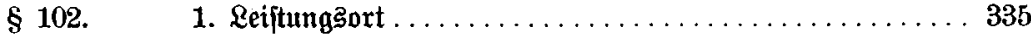

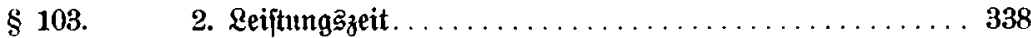

IV. Berzug.

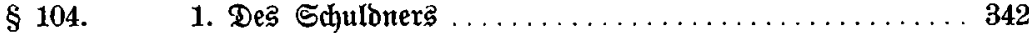

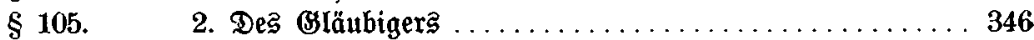

3weites Rapitel. (Elöjhen ber S屯uldverhältuiffe.

I. Untex Befriebigung bes Bläubigers.

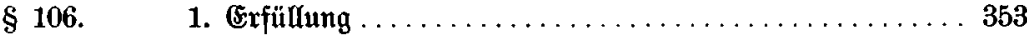

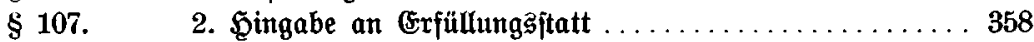

$\$ 108 . \quad 3$. \$interlegung $\ldots \ldots \ldots \ldots \ldots \ldots \ldots \ldots \ldots \ldots \ldots \ldots \ldots$

$\S 109 . \quad$ 4. $\mathfrak{U} u$ frechnung $\ldots \ldots \ldots \ldots \ldots \ldots \ldots \ldots \ldots \ldots \ldots \ldots \ldots \ldots$

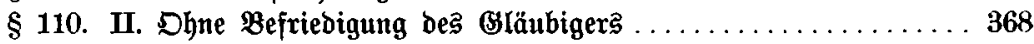

Drittes Rapitel. Die Subjefte ber Şuldverhältniffe.

I. $\mathfrak{B e c h j e l}$ berjelben.

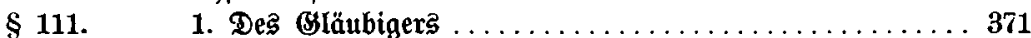

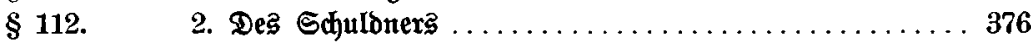


II. Mebrbeit berfelben.

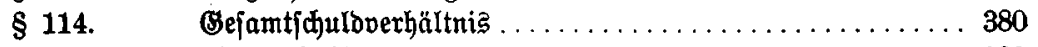

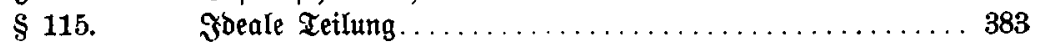

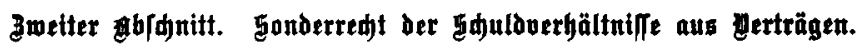

Erites Rapitel. Эm allgemeinen.

I. Einfđ̆ränfungen.

§ 116. $\quad$ Berbote. Bef. Spiel und $\mathfrak{B e t t e} \ldots \ldots \ldots \ldots \ldots \ldots \ldots 385$

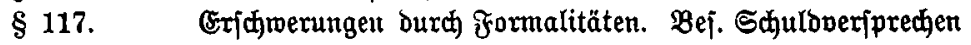

II. Beftärtungß̧mittel.

$\S 118 . \quad$ Draufgabe . . . . . . . . . . . . . . . . . . 392

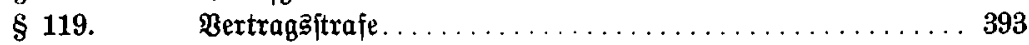

$\$$ 120. II. \&eiftung an Dritte $\ldots \ldots \ldots \ldots \ldots \ldots \ldots \ldots \ldots \ldots \ldots \ldots$

IV. Sonderrecht ber ๔丸ulbverbältniffe aus gegenjeitigen $\mathfrak{B e r t r a ̈ g e n . ~}$

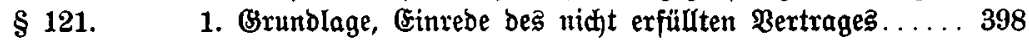

$\S 122 . \quad 2$. Rüftritt $\ldots \ldots \ldots \ldots \ldots \ldots \ldots \ldots \ldots \ldots \ldots \ldots \ldots \ldots$

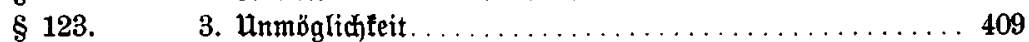

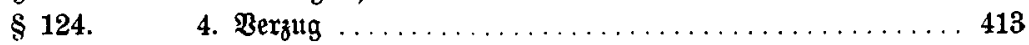

3weites Rapitel. Die einzelnen Shuldverhältriffe a $\mathfrak{z}$ Berträgen.

$\S$ 125. Cinteilung.

I. \$ejentlich gegenteitige Sđjulbverbältnifje.

1. Rauf.

$\S 126$.

a) Saçtauf.

$\S 127$.

$\mathfrak{I m}$ allgemeinen $\ldots \ldots \ldots \ldots \ldots \ldots \ldots \ldots \ldots \mathbf{4 1 7}$

$\S 128$.

Sejabr ........................ 423

$\S 129$.

Sewährleiftung wegen $\mathfrak{M a ̈ n g e l ~} \ldots \ldots \ldots \ldots \ldots 426$

b) $\mathfrak{f a u f}$ anderet Gegenftändo $\ldots \ldots \ldots \ldots \ldots \ldots \ldots .435$

$\$ 130 . \quad$ c) Befondere $\operatorname{Irten}$ des $\mathfrak{R a u f e z} \ldots \ldots \ldots \ldots \ldots \ldots 437$

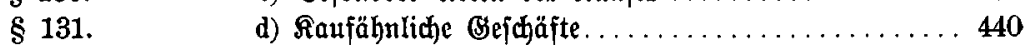

2. Miete und ßacht.

$\$ 132$.

$\S 133$.

a) Miete.

$\$ 134$.

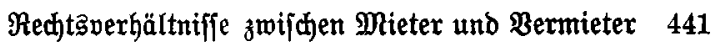

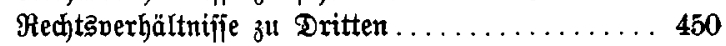

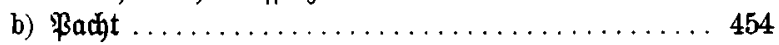

$\$ 135$.

3. Dienit = und Berfvertrag.

$\S 136$.

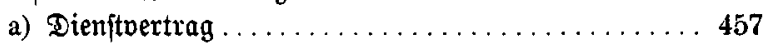

$\$ 137$.

$\S 138$.

$\S 139$.

b) ßerfvertrag.

c) Berwandte $\mathfrak{B e r t r a ̈ g e}$

467

\section{4. (Sejellichaft.}

$\S 140$.

ßerjönlidue Reijtungen.

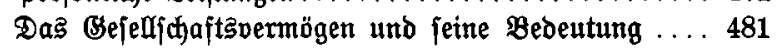

5. Bergleid. ......................... 489 


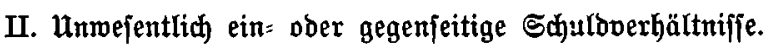

$\S 141 . \quad$ 1. Berwahrung $\ldots \ldots \ldots \ldots \ldots \ldots \ldots \ldots \ldots \ldots \ldots \ldots \ldots$

$\$ 142 . \quad 2$. Reibrente......................... 495

III. Einjeitige S丸uldoerbäItniffe nit untergeoroneter Gegenfeitigteit.

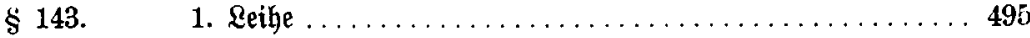

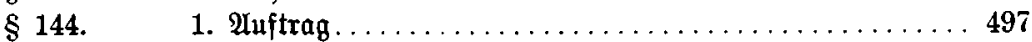

IV. Streng einjeitige Sđłulbverhältniffe.

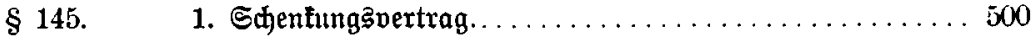

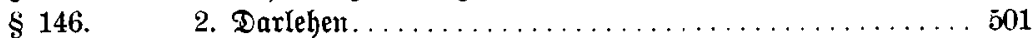

$\S 147 . \quad$ 3. Bürgidaft $\ldots \ldots \ldots \ldots \ldots \ldots \ldots \ldots \ldots \ldots \ldots \ldots$

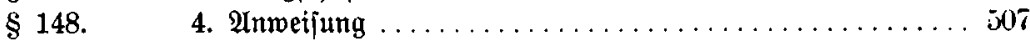

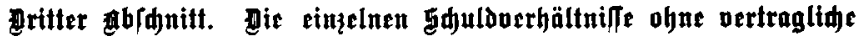
Begrüindung.

I. $\mathfrak{A}$ HE einjeitigem $\mathfrak{B e r j p r e c h e n . ~}$

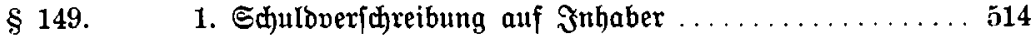

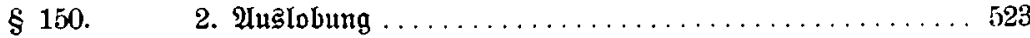

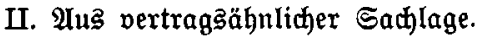

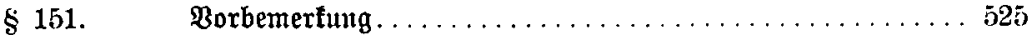

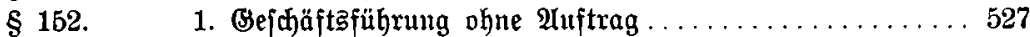

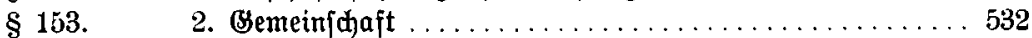

§ 154. 3. Borlegung von Sadjen $\ldots \ldots \ldots \ldots \ldots \ldots \ldots \ldots \ldots . \ldots \ldots 5$

4. Ungeredtfertigte Bereid)erung.

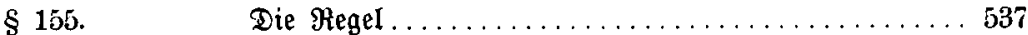

$\S 156 . \quad$ Einige Einzelfälle $\ldots \ldots \ldots \ldots \ldots \ldots \ldots \ldots \ldots \ldots 47$

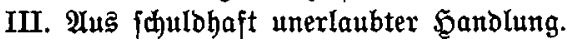

$\S 157 . \quad$ Borbemertung . . . . . . . . . . . . . . . . . . . 552

$\S 158$. Die brei allgemeinen Tatbeftände. . . . . . . . . . . . 554

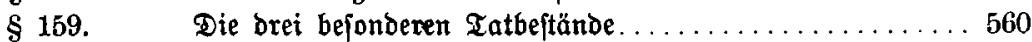

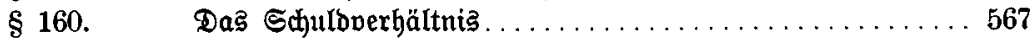

$\S 161$ IV. Âus fauldähnlider Sad)lage $\ldots \ldots \ldots \ldots \ldots \ldots \ldots \ldots \ldots 571$

Briffeg Burfy.

Gautienredit.

I. IUlgemeinhin.

Griler

$\S$ 162. Grunbbegriffe $\ldots \ldots \ldots \ldots \ldots \ldots \ldots \ldots \ldots \ldots \ldots \ldots 79$

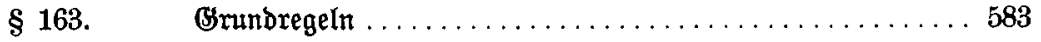

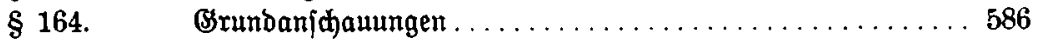

II. Das (Srumbbuctrecht.

1. Endgültige Eintragungen.

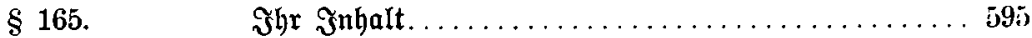

$\S 166 . \quad$ \hre Bornahme $\ldots \ldots \ldots \ldots \ldots \ldots \ldots \ldots \ldots \ldots$

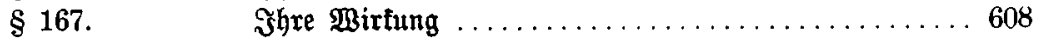

2. Porläufige (sintragungen.

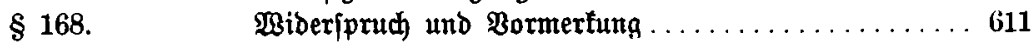

$\S 169 . \quad 3$. (Erfigung und $\mathfrak{B e r}$. 


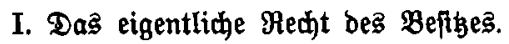

$\S 170 . \quad$ Erwerb und $\mathfrak{B e r l u f t}$ be马 $\mathfrak{B}$ Befibes . . . . . . . . . . . . . 621

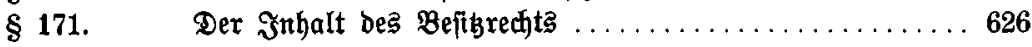

II. Arten und $\mathfrak{A b a r t e n}$ bes Befrigredts.

$\S 172 . \quad$ Der mittelbare $B$ efíb $\ldots \ldots \ldots \ldots \ldots \ldots \ldots \ldots \ldots \ldots, 631$

$\S 173 . \quad$ Sonftige $\mathfrak{A r t e n}$ bes Befizes....................634

I. Эnhalt bes Eigentumb. Breites Rapitel. Das Eigentum.

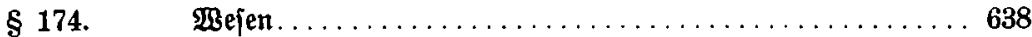

$\S 175 . \quad$ Radbarredt $\ldots \ldots \ldots \ldots \ldots \ldots \ldots \ldots \ldots \ldots \ldots, 642$

II. Errverb und Berluft des Eigentums.

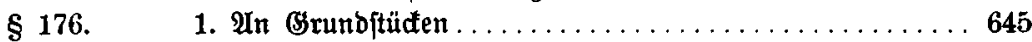

2. $\mathfrak{A n} \mathfrak{z} \mathfrak{a h r n i s . ~}$

$\S 177$.

$\S 178$.

$\S 179$.

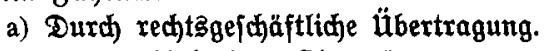

Bom bisherigen Eigentümer................647

$\mathfrak{B}_{\text {om }} \mathfrak{R i d} \mathrm{t}=$ (Eigentümer $\ldots \ldots \ldots \ldots \ldots \ldots \ldots 6 \mathbf{6 5 4}$

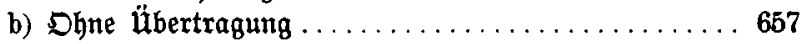

III. $2 \mathfrak{n}\{\mathfrak{p r u ̈ d}$ a aus bem cigentum.

1. Begen Borenthaltung.

$\S 180 . \quad$ Die rein binglithe Eeite....................6.668 6

\& 181. Die obligatorif

§ 182. Begenrechte wegen $\mathfrak{B}$ erwendungelt $\ldots \ldots \ldots \ldots \ldots, 676$

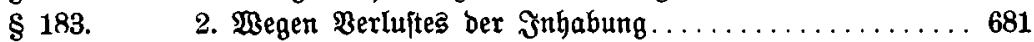

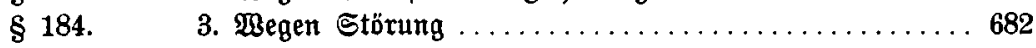

$\S 185$. IV. Miteigentum.................................6. 683

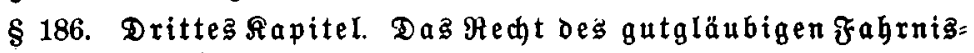
befigę................................. 685

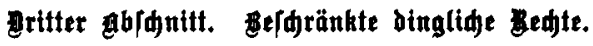

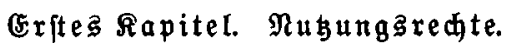

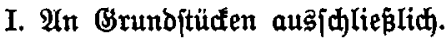

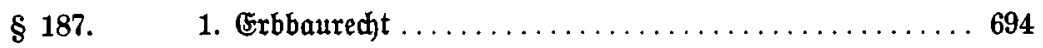

$\S 188 . \quad$ Begriff und $\mathfrak{F} 0$ lgen ...................65

§ 189. Die Brundbienttbarfeit mit „2Anlage“ bejonders ...... 701

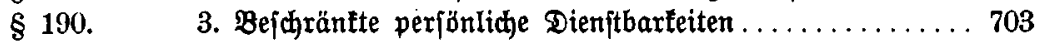
II. Riepbrauch.

1. $\mathfrak{A n}$ Sadhen.

$\S 191$.

$\S 192$.

a) Eigentliđer Saçnieß̧braudd.

$\S 193$.

Die allgemeinen Regeln ................. 705

Einige Einzelheiten ..................... 714

b) Uneigentlidjer ฐadnießbraud ............. 717

$\S 194$.

2. $\mathfrak{A n}$ Rectten.

a) $\mathfrak{\Im} \mathfrak{m}$ affgemeinen 


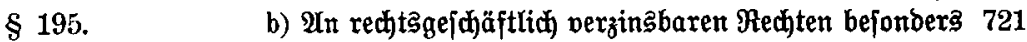

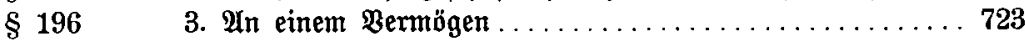

3weites Rapitel. Dinglidye Sఝuldoerhältuiffe.

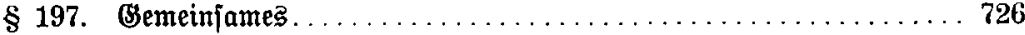

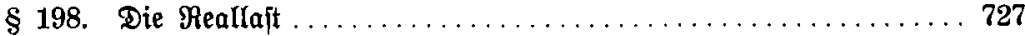

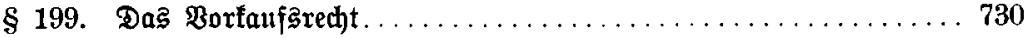

Drittes Rapiter. Berwertungarecte.

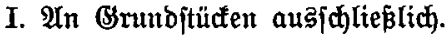

1. Allgemeines.

$\S 200$.

überblicf

$\S 201$.

Semeinjames

2. Die Bertehrshypothet.

a) Dhne Brief.

$\S 202$.

Berbältnis zwifdhen Đypotbef uno forberung . . . 749

$\S 203$.

$\S 204$. Die Cinzzlgeiten: Entftehung, Umfang und $\mathfrak{A} u \mathfrak{s}$ übbarfeit. . . . . . . . . . . . . . 754

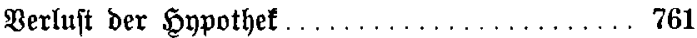

$\S 205$.

b) Mit $\mathfrak{B}$ rief $\ldots \ldots \ldots \ldots \ldots \ldots \ldots \ldots \ldots \ldots \ldots \ldots \ldots$

$\S 206 . \quad 3$. Die Sicherungahgpothef.................... 770

4. Wertgeminumgarechte.

$\S 207$.

(5)rundfhuld

Rentenifuld

$\S 209$.

II. $\mathfrak{A} \mathfrak{n}$ Fahrnis aușfhlieplich: Pfanbredyt.

1. Regelmäßige Beitaltung.

$\S 210$.

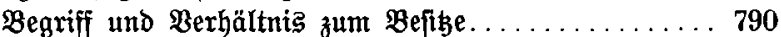

$\S 211$.

Einzelbeiten: $\mathfrak{B i s}$ zur $\mathfrak{A} u \mathfrak{s}$ übbarfeit.

$\S 212$.

(Einzelbeiten: $\mathfrak{A} u \mathfrak{s u ̈ b b a r f e i t ~ u n d ~} \mathscr{D u r c h}$ fübrung . . . . 804

$\S 213$.

$\S 214$.

$\S 215$.

(Einzelheiten: Das Erlöfchen bes Pfandrechts ...... 812

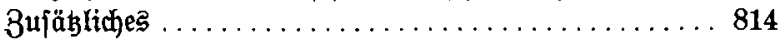

2. $\mathfrak{A} u$ ererorbentfiche Beftaltung $\ldots \ldots \ldots \ldots \ldots \ldots \ldots \ldots 815$

III. Pfandrecht an unförperlidjen (segenftänden.

$\S 216 . \quad \quad \Im m$ allgemeinen $\ldots \ldots \ldots \ldots \ldots \ldots \ldots \ldots \ldots \ldots \ldots . \ldots \ldots$

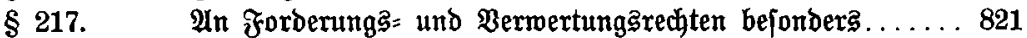

\section{Bierte Burfy.}

Jamilienredht.

Crefter ghidunitt. Cheredt.

§ 218. Einleitendes und $\mathfrak{B o r b e r e i t e n d e s . ~ D a ß ~ B e r l o ̈ b n i z ~}$

(Erites Rapitel. Ehelides Perjonenrect.

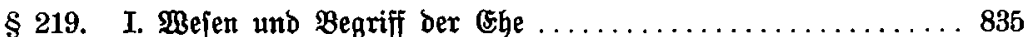

II. Eingehung Der Elye.

$\S 220$. 1. Grundbegriffe 
2. Die Ciuzelbeiten.

III. Mirfungen ber (she.

$\S 224 . \quad \ddot{u ̈ b e r w i e g e n d}$ perionentedtlich $\ldots \ldots \ldots \ldots \ldots \ldots \ldots \ldots 5$

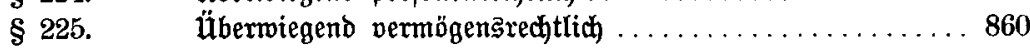

IV. A̛flö ung ber she.

§226. Grunblegendes $\ldots \ldots \ldots \ldots \ldots \ldots \ldots \ldots \ldots \ldots \ldots$

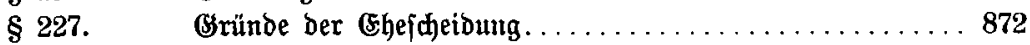

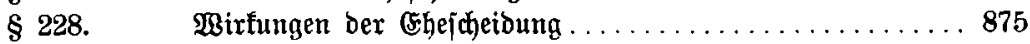

3reites Rapitel. Shelidges (süterredt.

I. Die (Strundlagen.

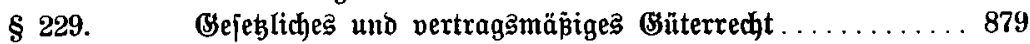

$\S 230$. Wirfung gegen Dritte. Süterrechtsregifter $\ldots \ldots \ldots \ldots .885$

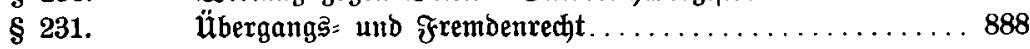

II. Die gejeblichen (süterrechte.

1. Da: (Süterrecht ber ebemäunliden Bermaltung und $\mathfrak{R u b =}$ niésung.

III. Die vertragßnmäßigen (Süterrechte.

1. Ullgemeine (Sütergemeinidaft.

$\S 236$.

Die $\mathfrak{B r u n b r e g e l n} \ldots \ldots \ldots \ldots \ldots \ldots \ldots \ldots \ldots \ldots . \ldots 11$

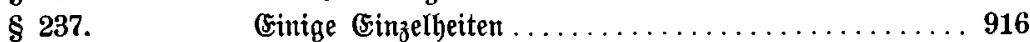

§ 238. Beendigung diejes (Suterftandes $\ldots \ldots \ldots \ldots \ldots \ldots . \ldots 918$

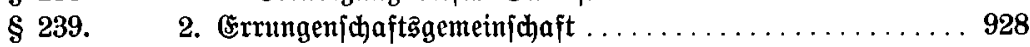

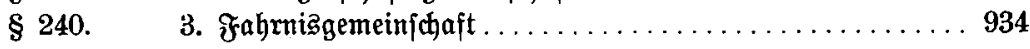

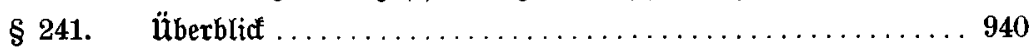

\section{Bmeiter ab/dnitt. Derwandt/d)aftsredt.}

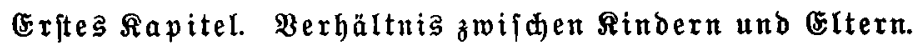

I. Birtliche, eheliche Rindidhaft.

$\S 242$. 1. Borausjebungen.

$\S 243$.

2. Mirfungen.

a) $\mathfrak{I}$ alfgemeinert

b) EIterlid)e (Semalt.

II. Wirfliche, auserebeliche Rindidaft.

§ 246. 1. Bei $\mathfrak{A b}$ tammung aủ nidtiger (she $\ldots \ldots \ldots \ldots \ldots 7$

2. Mangels $\mathfrak{A b j t a m m u n g ~ a u s ~ n i d h t i g e r ~ ( 5 h e . ~}$

$\S 247$ a) Shne Regitimation. 
b) Bei hinzutretender Regitimation.

§ 248. Durdh nadfolgende (5he $\ldots \ldots \ldots \ldots \ldots \ldots \ldots 973$

\$ 249.

$\S$ 250. III. Ânnahme an Rinbeş̧tatt $\ldots \ldots \ldots \ldots \ldots \ldots \ldots \ldots \ldots \ldots 978$

3weites Rapitel. Berbältnis grifhen Borfahren und $\mathfrak{A}$ b $\mathbf{f}$ ö m m lingent.

§ 251. Borauşebungen $\ldots \ldots \ldots \ldots \ldots \ldots \ldots \ldots \ldots \ldots \ldots \ldots .982$

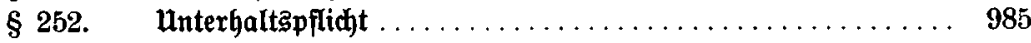

§ 253. Drittes Rapitel. Berwandtidaft unb ऽ山wägerf山aft im allgemeinen........................ 990

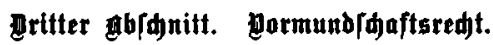

\$ 254. überbliaf und Grunbbegriffe $\ldots \ldots \ldots \ldots \ldots \ldots \ldots \ldots \ldots 996$

I. über Minberjährige.

Erites $\Re$ apitel. Bornunbjめaft.

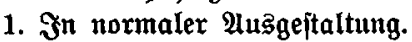

a) 2lnoronung ber Bormundfd)aft.

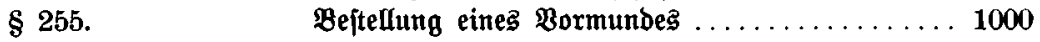

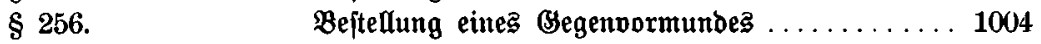

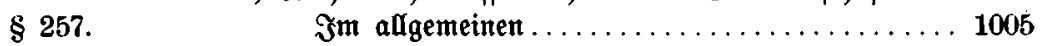

$\S 258 . \quad$ Cinige Ginzelheiten $\ldots \ldots \ldots \ldots \ldots \ldots \ldots \ldots 10 \ldots \ldots$

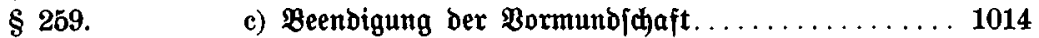

2. Befonbere $\mathfrak{A}$ iggejtaltungen.

$\S 260 . \quad$ Mehrere Mitoormünder . . . . . . . . . . . . . 1019

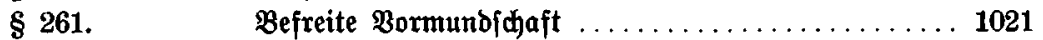

$\$ 262 . \quad$ Familienrat $\ldots \ldots \ldots \ldots \ldots \ldots \ldots \ldots \ldots \ldots \ldots \ldots$

$\S$ 263. II. über $\mathfrak{g o l j a ̈ h r i g e ~} \ldots \ldots \ldots \ldots \ldots \ldots \ldots \ldots \ldots \ldots \ldots \ldots \ldots$

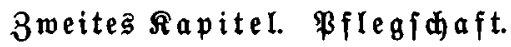

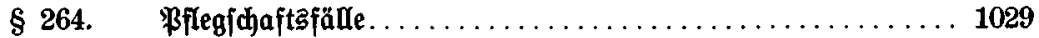

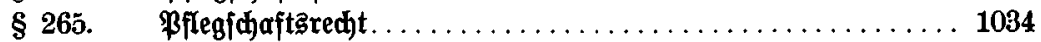

\section{Tünffe Budf.}

Gruxedit.

§266. Grundlegendes; Erbfall; äberblid. 1039

Grfter gb/dnitt. ofelaminadfolge von Tobes wegen.

Erites Rapitel. Exwerb ber (5rbja aft.

I. Die allgeneinen \&ehren.

1. (Erbfall, Berufung, Infall.

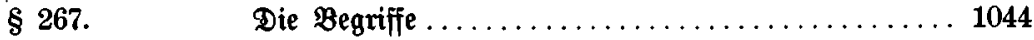

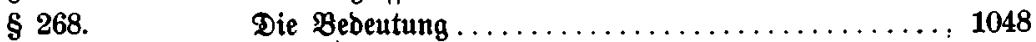

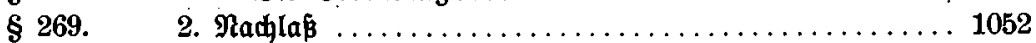

§ $270 . \quad 3$. Berufungsgrund, Berufungstatbeftand, Erbteil. . . . 1056 
II. Befeblid)e Crbjolge.

1. Bermandtenerbfolge.

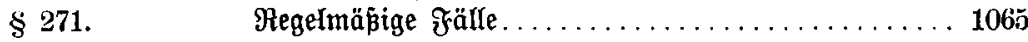

§ 272. Unregelmäßige $\mathfrak{F a ̈ l l e ~ \ldots \ldots \ldots \ldots \ldots \ldots \ldots \ldots \ldots \ldots . ~} 1073$

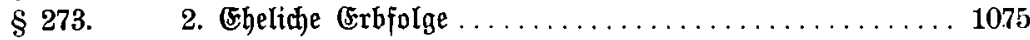

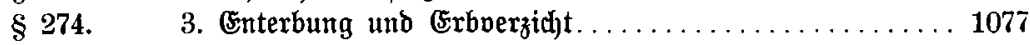

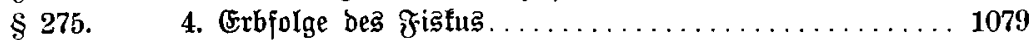

III. Gemillfüte Erbfolge.

1. Teptament.

$\S 276$.

a) (5rrichtung, Aufbebung, äupere Behanolung.

$\S 277$.

Teftierfähigłeit ...

Teftamentङerrid)tung . . . . . . . . . . . . . 1082

Ieftamentsaufhebung. . . . . . . . . . . . . 1087

$\S 278$.

$\S 279$.

Teftamenţeröffinung . ................. 1091

$\S 280$.

b) Bültigfeit und $\mathfrak{B i r t j a m f e i t ~} \ldots \ldots \ldots \ldots \ldots \ldots \ldots 1092$

$\S 281$.

c) Inhalt.

$\S 282$.

GSemöhnlidge Erbeinjegung . . . . . . . . . . . 1098

(srjagerbeinjebung. . . . . . . . . . . . . . 1102

$\S 283$.

\& 284.

Racherbeinfebung.

Sonftiger Эnhalt

$\S 285$.

d) Semeinj(haftlidyes Teftament

2. Erbvertrag.

\& 286.

$\Im \mathfrak{m}$ allgemeinen.

$\S 287$.

cinige bejondere fälle

1126

3weites Rapitel. Berluft ber (5rbfhaft.

§ 288. Borbemerfung

I. Xusfकlagung und Amrahme.

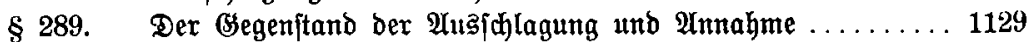

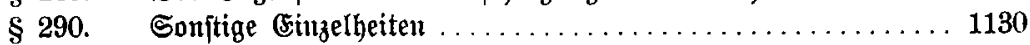

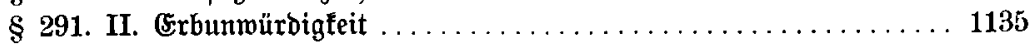

Drites Rapitel. Die Stellung Des Erben.

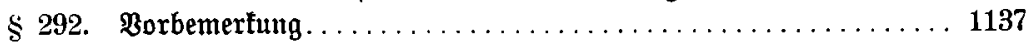

I. Die Stellung bes âleinerben.

1. Berechtigung.

$\S 293$.

a) $\mathfrak{U} u \mathfrak{S}$ ererbten $\mathfrak{A}$ (njprïchen.

$\S 294$. $\mathfrak{\Im m}$ allgemeinen $\ldots \ldots \ldots \ldots \ldots \ldots \ldots \ldots \ldots 1138$

$\S 295$.

Dex (Exbjhein inşbejondere.

b) (srbjchajtæanipruch).

$\S 296$.

2. Berpflichtungen.

Die (Sxundzüge und Subjette des $\mathfrak{A n}$ \{pruchs..... 1142

Der Begenftand und Segenanjprüche $\ldots \ldots \ldots \ldots 1146$

$\S 297$. überficht.

$\S 298$.

a) Iluffidiebende (sinreden ................ 1157

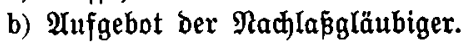

$\S 299$.

Die Mapregel 
c) Inventarerridtung

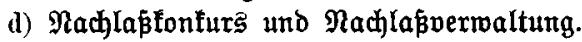

g) unbefđräntbare Şaftung.

II. Die Stelfung Der Mitexben.

1. 3ueinander.

$\S 309$.

a) Bor ber $\mathfrak{A}_{\mathfrak{L}}$ einanderjebing.

b) Die Auseinanderjebung.

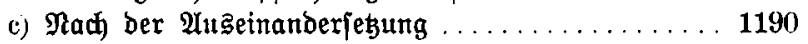

a) Beredtigung $\ldots \ldots \ldots \ldots \ldots \ldots \ldots \ldots \ldots \ldots \ldots 119 . \ldots \ldots$

b) Berpflidatungen.

Biertes $\mathfrak{A} a \mathfrak{p}$ itel. Teftamentzovllfteder.

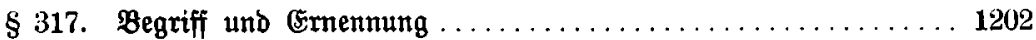

$\S$ 318. Die Stellung Des gemöhnIichen Teftamentకoollitrecfers. . . . . . 1206

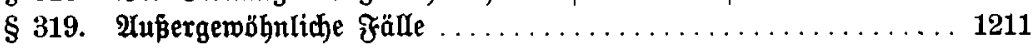

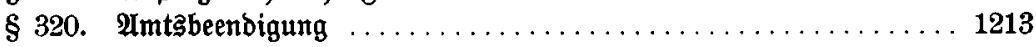

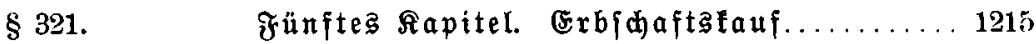

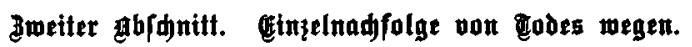

Erites Rapitel. Einzelverorbnungen don Tobes roegen.

I. Dur山 Berfügung des Erblafjers.

1. ßermä๘tniż. 


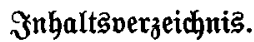

XIX

Gette ber

3reiteß אapitel. Bfliळtteilsredt.

ametten \$älfte

I. Sein Эnhalt.

1. Mein erbrectitlich.

$\S 330$.

a) Die Grunblagen

1238

$\S 331$.

b) Das Pflichtteil'srecht im eingelnen.

$\S 332$.

$\S 333$.

Die Birffamfeit3arten.

1243

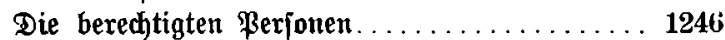

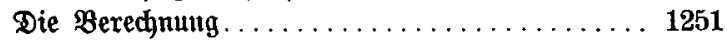

c) Der Bflidtteilsanfpruch bejonders̄.

$\S 334$.

Die Berpflichteten und die Raftabwälzang . . . . 1256

$\S 335$.

Sonjtige Cigentümlidłfeiten.

1259

2. Müdwixfjamfeit auf Berfügungen unter kebenben.

$\S 336$.

a) Betreffend Sdentungen.

b) Betreffeno $\mathfrak{A}$ (sjtattungen

II. Seine Contziebung oder Befđräntung.

$\S 338$.

Begen Berfehlung.

$\S 339$.

Эu guter $\mathfrak{A} \mathfrak{A} b$ ficht 